\title{
Masernelimination: Das Ziel ist in Reichweite
}

\author{
Virginie Masserey Spicher \\ Dr. med., Leiterin der Sektion Impfprogramme und Bekämpfungsmassnahmen, Bundesamt für Gesundheit
}

Das Jahr 2015 ist für die Elimination der Masern in der Schweiz entscheidend, und alle können für die Zielerreichung einen Beitrag leisten. Im Rahmen der breit abgestützten Nationalen Strategie zur Masernelimination 2011-2015 von Bund, Kantonen, medizinischen Fachorganisationen und Berufsverbänden wurden bereits viele Massnahmen umgesetzt - mit ermutigenden Resultaten.

\section{Die wichtigsten Ziele der Strategie}

1. dass in jeder neuen Geburtskohorte mindestens 95\% der zweijährigen Kinder mit 2 Dosen vor Masern geschützt sind,

2. dass bis Ende 2015 die Impflücken bei den nach 1963 Geborenen geschlossen sind und

3. dass Masern-Ausbrüche in allen Kantonen möglichst rasch mit einheitlichen Massnahmen unter Kontrolle gebracht werden.

\section{Die wichtigsten Massnahmen}

Das nationale Komitee für eine Schweiz ohne Masern mit 15 Persönlichkeiten aus Gesundheit, Sport, UNICEF, Konsumentenschutz und Politik setzt sich auf politischer Ebene und mittels Medienarbeit für die breite Unterstützung der Masernelimination ein.

Eltern spricht man über verschiedene Kanäle auf die Wichtigkeit, ihr Kind rechtzeitig impfen zu lassen, und die Konsequenzen des Nicht-Impfens an. Im Fall eines

\section{Impfempfehlungen}

- Als Basisimpfung für alle Kinder empfiehlt das Bundesamt für Gesundheit (BAG) die kombinierte Impfung gegen Masern, Röteln und Mumps (MMR): die erste Dosis im Alter von 12 Monaten, die zweite zwischen 15 und 24 Monaten.

- Eine Nachholimpfung (MMR) ist in jedem Alter möglich und wird allen nach 1963 geborenen Personen empfohlen, die nicht zweimal geimpft sind und die Masern noch nicht hatten.

- Für Säuglinge mit erhöhtem Risiko einer Masernerkrankung (Frühgeborene, in Krippen, bei Tagesmüttern) oder bei einer Epidemie ist die erste Dosis MMR mit 9 Monaten und die zweite im Alter von 12 bis 15 Monaten empfohlen. Bei direktem Kontakt mit einer erkrankten Person sollte eine erste Dosis bereits ab 6 Monaten verabreicht werden.

- Der Mindestabstand zwischen zwei Dosen beträgt jeweils einen Monat.
Masernausbruchs können ungeimpfte Kinder vorübergehend aus der Kita oder Schule ausgeschlossen werden. Dazu versenden die kantonalen Gesundheitsbehörden z.B. persönliche Briefe, und beim Krippenund Schuleintritt gibt es spezifische Informationen. Kinderarztpraxen vereinbaren bei einer Konsultation mit den Eltern von Kleinkindern gleich einen Termin zum empfohlenen Zeitpunkt der MMR-Impfung oder laden aktiv dazu ein.

Das elektronische Impfbüchlein für alle wird gefördert. Man kann es via App oder auf www.meineimpfungen. ch selbstständig oder durch eine medizinische Fachperson erstellen, bei Säuglingen idealerweise gleich ab den ersten Impfungen in der (Kinder-)Arztpraxis. Es erinnert automatisch an allenfalls fehlende Impfungen, ist passwortgeschützt jederzeit abrufbar, geht nicht mehr verloren und kann ausgedruckt werden. Sobald es durch einen Arzt oder Apotheker validiert ist, hat es die gleiche Gültigkeit wie ein Impfausweis aus Papier.

Nachholimpfungen mit maximal 2 Dosen benötigen insbesondere Jugendliche und bis 50-jährige Erwachsene, die häufig nicht wissen, dass sie nicht oder nur ungenügend geschützt sind. Dazu führen Bund und Kantone seit 2013 gemeinsam die nationale Kampagne «Stopp Masern» (www.stopmasern.ch) durch. Es geht vor allem darum, Jugendliche und Erwachsene dazu zu motivieren, ihren Impfstatus kontrollieren zu lassen und allfällige Impflücken so rasch wie möglich mit einer Nachholimpfung zu schliessen. Diese ist bis Ende 2015 von der Franchise befreit, und in vielen Kantonen fördern Aktionen vor Ort die Nachholimpfung. Ärztinnen und Ärzte überprüfen systematisch die Impfausweise und führen die nötigen Nachholimpfungen durch. Eine Hochrechnung aufgrund einer Erhebung in Praxen der ärztlichen Grundversorgung des Sentinella-Meldesystems ergab für 2014 insgesamt 33500 Nachholimpfungen bei 2- bis 50-Jährigen. Die Befragten gaben an, dass sie in 10 Monaten durchschnittlich 4,4 Nachholimpfdosen verabreichten (Allgemeinärzte und Internisten: 3,5 Dosen, Kinderärzte: 9,1 Dosen). Ein Internist verabreichte sogar 109 und ein Kinderarzt 107 Dosen. 
Die 2013 publizierten «Richtlinien zur Bekämpfung von Masernausbrüchen" geben einen Rahmen, wie in allen Kantonen Maserninfektionen rasch und effizient unter Kontrolle gebracht werden können.

\section{Aktueller Stand}

In praktisch allen Kantonen und Altersklassen nahm die Durchimpfung weiter $z u$. In den acht Kantonen, die an der Erhebung 2014 teilnahmen, sind bereits 87\% der zweijährigen Kinder mit zwei Impfdosen vor Masern geschützt. Besonders erfreulich: Gerade in Kantonen, die bislang unter dem nationalen Durchschnitt lagen, gab es deutliche Fortschritte: zum Beispiel in Appenzell-Innerrhoden von 50 auf 85\%, in Schwyz von 76 auf $82 \%$ und in Luzern von 82 auf $87 \%$. Genf, Waadt und Freiburg haben bei den 8-Jährigen Schulkindern die angestrebten $95 \%$ bereits erreicht. Ausserdem ist Genf der erste Kanton, in dem über 95\% der 2-Jährigen mit zwei Dosen geschützt sind. Damit Masern als eliminiert gelten, müssen 95\% der Bevölkerung immun sein, und jährlich darf noch maximal eine Erkrankung pro Million Einwohner vorkommen.

Die Anzahl bestätigter Masernfälle ging in der Schweiz in den letzten Jahren zurück: von $664 \mathrm{im} \mathrm{Jahr} \mathrm{2011,} \mathrm{über}$ $65 \mathrm{im} 2012$ und $176 \mathrm{im} 2013$ bis auf noch $23 \mathrm{im}$ 2014. Dies entsprach 2014 einer Inzidenz von 2,8 Erkrankungen pro Million Einwohner. Somit ist dieses Kriterium für die Elimination noch nicht ganz erreicht. Die Krankheit tritt vorwiegend noch bei Jugendlichen und Erwachsenen ohne genügenden Impfschutz auf: Im Zeitraum von 2012 bis 2014 lag das mittlere Alter der Patienten bei 15 Jahren, die Tendenz setzte sich 2015 fort.

Dr. med.

Virginie Masserey Spicher Eidgenössisches

Departement des Innern Bundesamt für Gesundheit Schwarzenburgstrasse 157 CH-3003 Bern virginie.masserey[at] bag.admin.ch
In der WHO-Region Europa sind derzeit 50\% der Länder masernfrei. 2014 traten jedoch noch über $16000 \mathrm{Ma}$ sernfälle auf, mit Ausbrüchen in Italien, Slowenien, Bosnien und Deutschland. In Berlin starb im Februar 2015 ein nicht geimpftes, erst 18 Monate altes Kind an Masern. Mit Reisenden gelangen Masernviren auch in be-

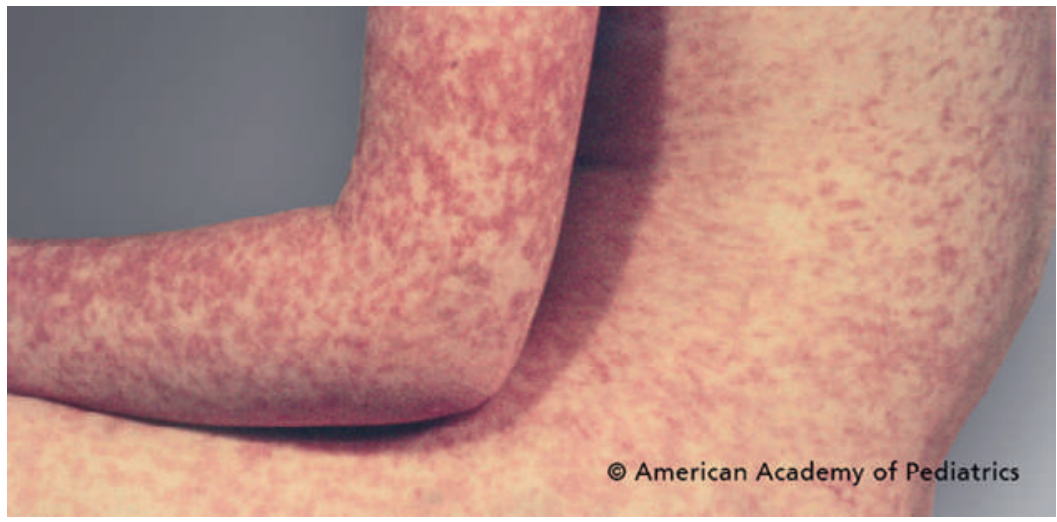

Die Masern sind nicht speziell eine Kinderkrankheit: Sie können in jedem Alter auftreten.
Wie können Gesundheitsfachpersonen dazu beitragen, die Masern in der Schweiz zu eliminieren?

1. Über Masern sprechen: Dank der Impfung ist die Krankheit mittlerweile so selten, dass man die teils schwersten Komplikationen kaum noch kennt. Es gilt, die bei einigen Personen bestehenden Ängste vor Impfnebenwirkungen ernst zu nehmen, Verunsicherungen anzusprechen und durch sachliche Information das Vertrauen in die Impfempfehlungen zu stärken.

2. Masernerkrankungen vorbeugen: Jeder Arzt-Patienten-Kontakt (z.B. in der Notfallstation, vor Auslandreisen, bei gynäkologischen Kontrollen) kann dazu genutzt werden, im Impfausweis aller nach 1963 geborenen Patientinnen und Patienten den Masern-Impfstatus zu überprüfen und fehlende Impfungen sofort oder beim nächsten Termin nachzuholen. Ausserdem sollten alle Fachpersonen im Medizinalbereich sowie in der Säuglingsbetreuung auch selbst über einen Impfschutz verfügen.

3. An Masern denken: Jeder Verdachtsfall (Trias: 1. Fieber, 2. makulopapulöses Exanthem, 3. Husten, Rhinitis oder Konjunktivitis) soll sofort an den kantonsärztlichen Dienst gemeldet und per Laboranalysen auf Masern getestet werden.

reits masernfreie Länder. So kam es 2014 zu Ausbrüchen in Brasilien, und Anfang 2015 erkrankten in den USA, ausgehend von einem Masernfall im Disneyland, mehr als 100 meist nicht-geimpfte Kinder und Erwachsene. Das Ziel einer masernfreien Schweiz rückt in greifbare Nähe. Gegenwärtig sind Masernausbrüche aber immer noch jederzeit möglich. Das heisst, es lohnt sich weiter zu handeln. Die Impfung ist eine einfache, sichere, wirksame und nicht zuletzt auch kostensparende Gesundheitsinvestition für alle.

Am Freitag, 6. November, findet (gleichzeitig mit dem Grippeimpftag) der «Stopp-Masern-Tag» statt. Noch bis Ende 2015 ist die Masernimpfung für alle, die sich vor der Krankheit schützen möchten, von der Krankenkassen-Franchise befreit. Es lohnt sich also besonders jetzt, seine Impflücken zu schliessen.

\section{Weitere Informationen}

- www.stopmasern.ch: Webseite mit Infos und Abbildungen zu den Masern, dem Online Risiko-Check sowie zur Kampagne inkl. Bestellmöglichkeiten für Kampagnenmaterial und zu Aktionen in den Kantonen.

- www.bag.admin.ch/masern: Webseite des BAG mit aktuellen Zahlen und vielen Informationen zur Krankheit, Impfung und Strategie sowie Faktenblättern für die Bevölkerung und Fachpersonen.

- www.meineimpfungen.ch: Gratis sein persönliches elektronisches Impfbüchlein erstellen: Es ist passwortgeschützt abrufbar, erinnert automatisch an Impfungen - und kann nie mehr verloren gehen. 